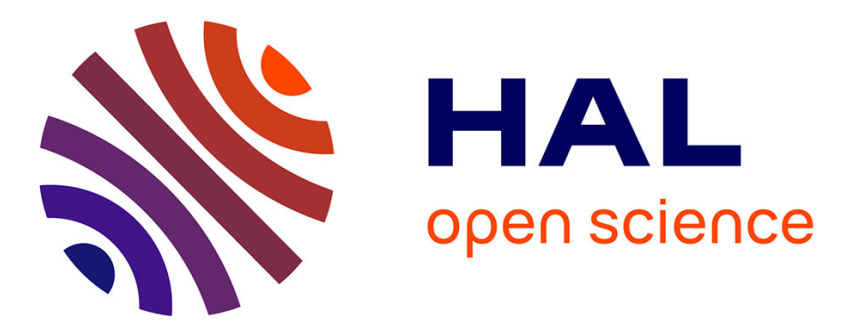

\title{
Observation of backaction-evading measurement of an optical intensity in a three-level atomic nonlinear system
}

Philippe Grangier, Jean-François Roch, Gérard Roger

\section{To cite this version:}

Philippe Grangier, Jean-François Roch, Gérard Roger. Observation of backaction-evading measurement of an optical intensity in a three-level atomic nonlinear system. Physical Review Letters, 1991, 66, pp.1418-1421. 10.1103/PhysRevLett.66.1418 . hal-00559097

\section{HAL Id: hal-00559097 \\ https://hal-iogs.archives-ouvertes.fr/hal-00559097}

Submitted on 23 May 2016

HAL is a multi-disciplinary open access archive for the deposit and dissemination of scientific research documents, whether they are published or not. The documents may come from teaching and research institutions in France or abroad, or from public or private research centers.
L'archive ouverte pluridisciplinaire HAL, est destinée au dépôt et à la diffusion de documents scientifiques de niveau recherche, publiés ou non, émanant des établissements d'enseignement et de recherche français ou étrangers, des laboratoires publics ou privés. 


\title{
Observation of Backaction-Evading Measurement of an Optical Intensity in a Three-Level Atomic Nonlinear System
}

\author{
Philippe Grangier, Jean-François Roch, and Gérard Roger \\ Institut d'Optique, BP 147, F91403 Orsay CEDEX, France
}

(Received 23 October 1990)

\begin{abstract}
Backaction-evading measurement of an optical intensity has been observed, using a three-level system in a sodium atomic beam as a nonlinear medium. The principle of this experiment is to use the crossphase-modulation effect, which in principle allows one to read nondestructively the quantum intensity fluctuations of the "signal" beam when detecting the phase fluctuations of the "meter" beam. Using a cavity-enhancement scheme, the quantum noise measured when recombining the signal and meter photocurrents is $0.8 \mathrm{~dB}$ below the shot-noise level.
\end{abstract}

PACS numbers: 42.50.Dv, 03.65. Bz, 32.80.Wr

It has been known since the beginning of quantum mechanics that a quantum measurement usually perturbs the quantity which is measured, adding "backaction noise" to the system under study. " A well-known system is the harmonic oscillator: In this case, it was shown that "quantum-nondemolition" (QND) measurements can be performed; i.e., it is possible to leave the observed quadrature component unchanged, and to add the backaction noise to the other (complementary) quadrature component. ${ }^{2}$ Such measurements were implemented in the optical domain, ${ }^{3,4}$ where the harmonic oscillator is a mode of the electromagnetic field, and where the coupling responsible for the measurement is created using either $\chi^{(2)}$ or $\chi^{(3)}$ optical nonlinearities. ${ }^{5,6} \mathrm{We}$ recently proposed ${ }^{7,8}$ to perform QND measurements using nearly resonant two-photon nonlinearities, ${ }^{9-14}$ in atomic three-level systems excited by two different (initially independant) laser beams. The basic physics of this nonlinear coupling can be understood very simply as a crossphase-modulation effect: In the lowest-order $\chi^{(3)}$ approximation, the refractive index for one beam will depend on the intensity of the other beam, while the usual self-phase-modulation (Kerr effect) can be made much smaller. It can be shown ${ }^{7,8,15}$ that this coupling directly yields QND measurements of the intensity fluctuations of a "signal" beam, which are copied into phase fluctuations of a "meter" beam by the measurement process. The measured quantity (intensity fluctuations) is left unchanged, while the backaction noise appears on the phase fluctuations of the signal beam.

In this Letter we report the experimental implementation of a QND measurement scheme using two-photon nonlinearities in an atomic three-level system. The overall experimental setup is shown in Fig. 1. The levels involved consist of the $3 s_{1 / 2}-3 p_{3 / 2}-3 d_{5 / 2}$ atomic cascade in sodium. Two light beams at the required wavelengths of 589.0 and $819.5 \mathrm{~nm}$ (ir) are obtained from two cw electronically stabilized dye lasers, pumped by a single argon-ion laser. A sodium atomic beam provides a density of $5 \times 10^{11}$ atoms $/ \mathrm{cm}^{3}$, with a Doppler width of about $200 \mathrm{MHz}$ FWHM. The interaction region with the laser beams is $1 \mathrm{~cm}$ long. The atomic beam is surrounded by a single-ended optical cavity $(8 \mathrm{~cm}$ long, 5 $\mathrm{cm}$ mirror radius), which resonates for the ir laser light exciting the upper transition. The transmittivity of the input-output mirror is $t^{2}=0.006$, while total losses are $p^{2}=0.011$, including both mirror transmittivities. The light beams inside the cavity are circularly polarized to optimize the nonlinear coupling, and the output ir beam is separated from the input beam using a prism polarizer. Both beams are detected using $p-i-n$ silicon photodiodes, followed by low-noise preamplifiers. ${ }^{16}$ The photocurrents are added or subtracted using $0^{\circ}-180^{\circ}$ power combiners, and sent to a spectrum analyzer. When taking data, the spectrum analyzer is set to zero frequency span, and a noise analysis frequency is selected. ${ }^{17}$ Then, the noise of the photocurrents is analyzed while the optical cavity is swept onto resonance of a noise sideband

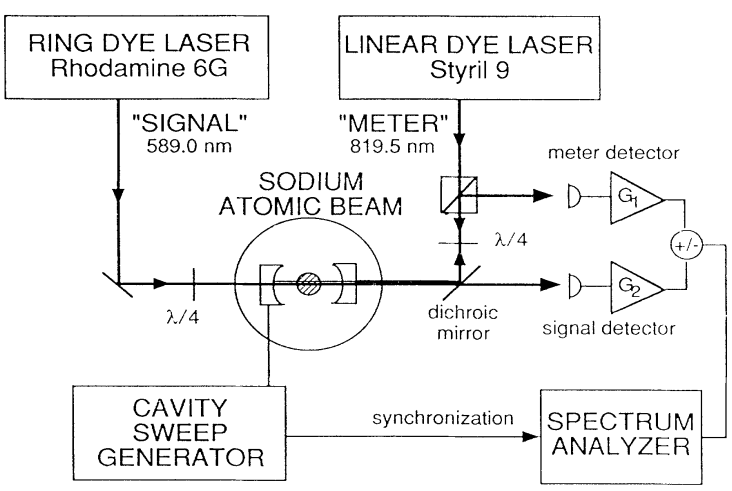

FIG. 1. Experimental setup. Two lasers at $589.0 \mathrm{~nm}$ ("signal" beam) and $819.5 \mathrm{~nm}$ ("meter" beam) are tuned close to the resonances of the $3 s_{1 / 2}-3 p_{3 / 2}-3 d_{5 / 2}$ cascade in a sodium atomic beam. The single-ended optical cavity resonates for a noise sideband of the meter beam. The quantum intensity noises of the output light beams are correlated, while the quantum intensity noise of the signal beam is not changed, providing evidence for a QND measurement. 
(NSB) of the ir beam, corresponding to the frequency which has been set on the spectrum analyzer. More precisely, this NSB resonance is obtained when the cavity is detuned from the laser frequency by an amount equal to the noise frequency set on the spectrum analyzer. ${ }^{17}$ The optical cavity has two roles: First, it enhances the nonlinear coupling (see below), and second, it turns the initial readout phase modulation of the meter beam into an intensity modulation, which can be directly detected. ${ }^{5,15,18}$

Typical results are shown in Fig. 2, where all noise levels refer to noise powers for the fluctuations of the photocurrents. Curves $a$ and $b$ show the separate noise levels of the probe and meter beams. When the NSB resonance is achieved, the noise of the meter beam is increased, while the noise of the signal beam is left unchanged. Outside this resonance, both noise levels have been checked to be at the shot-noise level. They are adjusted to the same electrical level by slight tuning of the preamplifiers gains. Curves $c$ and $d$ show the recombined photocurrents. Outside the resonance, the noises are obviously uncorrelated, and the noise level increases by $3 \mathrm{~dB}$, independently of the relative recombination phase. On the other hand, the noise of the meter beam
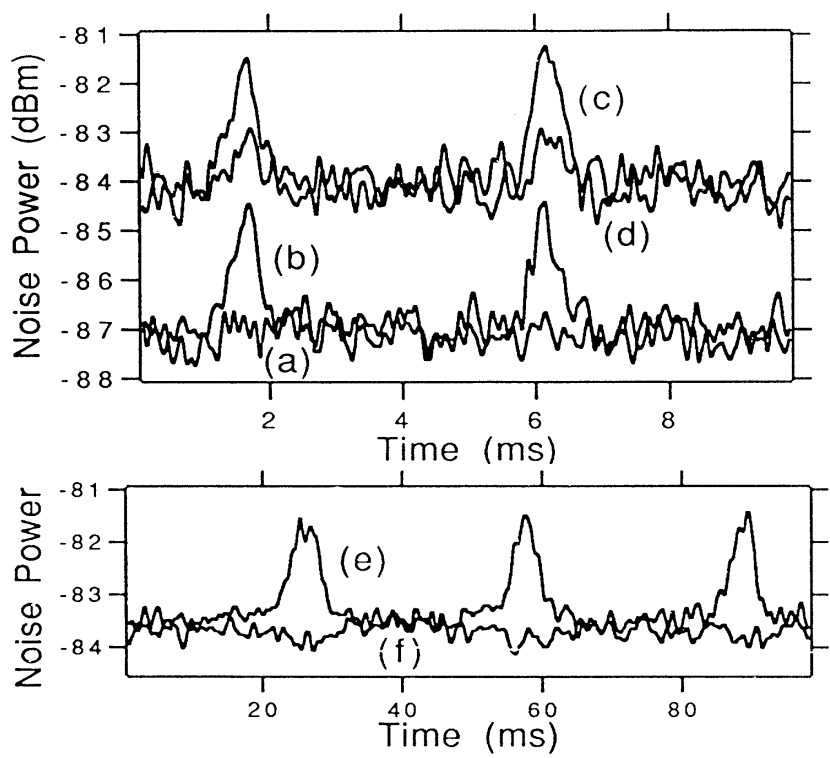

FIG. 2. Noise levels recorded while the cavity is swept over a noise sideband of the meter beam. The spectrum analyzer is set to zero-frequency span, $14-\mathrm{MHz}$ center frequency, $100-$ $\mathrm{kHz}$ radio-frequency bandwidth, $300-\mathrm{Hz}$ video bandwidth. Curves $a$ and $b$ are respectively the signal and meter noises, recorded separately. Other curves are the minimum and maximum noise levels of the combined photocurrents, obtained by adding or subtracting the signal and meter photocurrents. Curves $c$ and $d$ correspond to a one-photon detuning $\Delta=-1.75$ $\mathrm{GHz}$ and a two-photon detuning $\delta=-1 \mathrm{GHz}$, while curves $e$ and $f$ are obtained for $\Delta=+2.25 \mathrm{GHz}$ and $\delta=-1 \mathrm{GHz}$, with a cavity sweep 10 times slower (video bandwidth $30 \mathrm{~Hz}$ ). at the NSB resonance is correlated with the (unchanged) noise of the signal beam: Adding or subtracting the photocurrents results in a $1.5-\mathrm{dB}$ change of the noise levels. For this experiment, both laser intensities on the atoms were close to $10 \mathrm{~W} / \mathrm{cm}^{2}$ ( $1 \mathrm{~mW}$ power). The one- and two-photon detunings were respectively $\Delta=-1.75 \mathrm{GHz}$ and $\delta=-1 \mathrm{GHz}$, relative to the $F=2$ ground-state hyperfine sublevel. Other experiments were done for an opposite sign of the one-photon detuning, i.e., $\Delta=+2.25$ $\mathrm{GHz}$ and $\delta=-1 \mathrm{GHz}$, keeping the other parameters at the same values. In the latter case, the correlations were clearly increased, to about $2.5 \mathrm{~dB}$ (see curves $e$ and $f$ ). Moreover, the differenced noise was below the shot-noise level of the recombined photocurrent, by about $0.4 \mathrm{~dB}$. This shows clearly the quantum character of the correlation established between the signal and meter beams. ${ }^{19}$ These results could be further improved by optimizing the electronic gain of the meter channel. ${ }^{5,6,18,20}$ The combined photocurrents of the signal and attenuated meter are shown in Fig. 3. These curves are corrected for the amplifier noises, and normalized so that $0 \mathrm{~dB}$ is the shot-noise level of the signal alone. The differenced noise is now $0.8 \mathrm{~dB}$ below the shot-noise level of the recombined photocurrent, and reaches the shot-noise level of the signal alone.

One important advantage of this scheme is that only direct detection is needed, but we also used a homodyne detection setup, ${ }^{6}$ in order to check qualitatively that the signal phase noise is increased when the measurement is performed. On the other hand, the output-signal intensity fluctuations remain at the shot-noise level, within our experimental precision. Therefore, the intensity noise added to the signal beam, if any, is more than $10 \mathrm{~dB}$ below the shot-noise level. This is a good indication that the QND condition is satisfied. However, we notice that

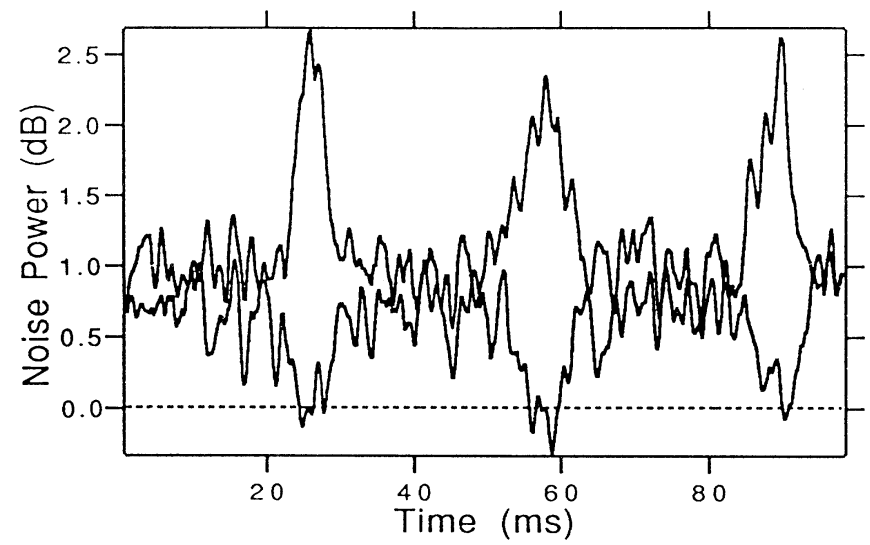

FIG. 3. Combined noise levels with 6-dB attenuation of the meter photocurrent. These curves are corrected for the amplifier noises, and normalized so that $0 \mathrm{~dB}$ corresponds to the shot-noise level of the signal alone. The differenced photocurrent goes $0.8 \mathrm{~dB}$ below the combined shot-noise level. 
all experiments to date ${ }^{5,6}$ including this one, check only the "state preparation" properties of the device, ${ }^{14,21}$ i.e., the fluctuations and correlations of the output beams. A true assessment of the QND properties should involve some characterization of the correlations between the input and the output fluctuations, using, for instance, two QND devices following each other. This will be the subject of further work.

The measured quantities are the variances (i.e., noise fluctuation powers), of the signal, meter, and recombined photocurrents, which will be denoted, respectively, $V_{s}^{\text {out }}$, $V_{m}^{\text {out }}$, and $V_{\varepsilon}^{\text {out }}(\varepsilon= \pm 1$ depending on the recombination phase). Assuming that the shot-noise levels of both beams have the conventional value of 1 , and correcting for the amplifier noises, one obtains $\mathbf{s}^{7,8,15}$

$$
\begin{aligned}
& V_{s}^{\text {out }}=1, \\
& V_{m}^{\text {out }}=1+\eta_{m} N_{m}, \\
& V_{\varepsilon}^{\text {out }}=2+\eta_{m} N_{m}+2 \varepsilon C_{m s}\left(\eta_{m} \eta_{s}\right)^{1 / 2},
\end{aligned}
$$

where $N_{m}$ and $C_{m s}$ are respectively the meter excess noise and the correlation between the meter and the signal, introduced by the QND device. Here, $C_{m s}$ is defined as the average product of the meter and signal intensity fluctations. The parameters $\eta_{s}$ and $\eta_{m}$ (with $\eta_{s} \cong \eta_{m}$ $\cong 0.5$ ) are the overall detection efficiencies of the signal and meter channels, including the linear cavity losses. According to our experimental results, we will neglect any excess noise added to the signal beam. For both negative $(n)$ and positive $(p)$ values of the detuning $\Delta$, the experimental values corresponding to the results of Fig. 2 are

$$
\begin{aligned}
& N_{m}^{p}=1.5, \quad C_{m s}^{p}=0.9, \\
& N_{m}^{n}=3, \quad C_{m s}^{n}=0.7,
\end{aligned}
$$

where a conservative estimate of the experimental uncertainties is $20 \%$ on all values.

The two-photon cross-phase-modulation effect can be calculated to the lowest $\chi^{(3)}$ approximation by solving the optical Bloch equations for a three-level system. ${ }^{22}$ We will consider only purely radiative decay, and denote by $\gamma_{1}, \gamma_{2}$ the intermediate- and upper-level transverse linewidths (half of the Einstein coefficients). The atomlaser detunings and Rabi frequencies will be denoted respectively $\delta_{1}, \Omega_{1}$ (lower transition) and $\delta_{2}, \Omega_{2}$ (upper transition). $\Delta=\delta_{1}$ is the one-photon detuning, and $\delta=\delta_{1}$ $+\delta_{2}$ is the two-photon detuning. All detunings are much larger than $\gamma_{1}, \gamma_{2}$, and the Doppler width (dispersive limit). Finally, the dipole matrix elements are $d_{1}, d_{2}$. Assuming that $\Delta \gg \delta$, the lowest-order effect of the laser fields is to induce a coherence between the ground and upper levels, whose expression is simply $\Omega_{1} \Omega_{2} / \Delta \delta$. The resulting nonlinear polarizations for the lower and upper transitions are then respectively $n_{\mathrm{at}} d_{1} \Omega_{1} \Omega_{2}^{2} / \delta \Delta^{2}$ and $n_{\text {at }} d_{2} \Omega_{2} \Omega_{1}^{2} / \delta \Delta^{2}$, where $n_{\text {at }}$ is the atomic number density.
These polarizations translate into nonlinear phase shifts for the laser fields, with respective values $n_{\mathrm{at}} k_{1} L d_{1}^{2} \Omega_{2}^{2} /$ $2 \varepsilon_{0} \hbar \delta \Delta^{2}$ and $n_{\text {at }} k_{2} L d_{2}^{2} \Omega_{1}^{2} / 2 \varepsilon_{0} \hbar \delta \Delta^{2}$. Here, $k_{1}=2 \pi / \lambda_{1}$ and $k_{2}=2 \pi / \lambda_{2}$ are the wave vectors of the laser fields, and $L$ is the total path length through the nonlinear medium. Using the definitions of $\gamma_{1}, \gamma_{2}$, one obtains convenient final forms for the nonlinear phase shifts:

$$
\begin{aligned}
& \kappa_{1}=n_{\text {at }} L\left(3 \lambda_{1}^{2} / 4 \pi\right) \gamma_{1} \Omega_{2}^{2} / \delta \Delta^{2}, \\
& \kappa_{2}=n_{\text {at }} L\left(3 \lambda_{2}^{2} / 4 \pi\right) \gamma_{2} \Omega_{1}^{2} / \delta \Delta^{2} .
\end{aligned}
$$

We note that, though they look nonsymmetric, $\kappa_{1}$ and $\kappa_{2}$ are equal if the field intensities are the same in photons units. The value of the nonlinear refractive index can then be inserted into a calculation of the fluctuations using the semiclassical linear input-output technique. ${ }^{23}$ At the NSB resonance in our linear cavity setup, the predicted value ${ }^{15}$ of the correlation coefficient $C_{m s}$ is $(2 / p)\left(\kappa_{1} \kappa_{2}\right)^{1 / 2}$, where $p^{2}$ has been previously defined, while the noise coefficient $N_{m}$ is $(2 / p)^{2} \kappa_{1} \kappa_{2}$. The signature of an "ideal" nonlinear effect ("parametric" limit) lies in the relation $N_{m}=C_{m s}^{2}$.

Typical experimental values (in $\gamma_{1}$ units) are $\gamma_{2}=0.8$, $\Omega_{1}=\Omega_{2}=30, \Delta=350, \delta=200$, and $n_{\mathrm{at}} L 3 \lambda_{1} \lambda_{2} / 4 \pi=1200$, so that $C_{m s}=0.8$, which agrees fairly well with the observed values. The complete calculation, including the sodium hyperfine levels and polarization effects, does not significantly change the above value of $C_{m s}$. However, this simple theory fails to explain the experimentally observed deviations from the relation $N_{m}=C_{m s}^{2}$, which clearly depend on the sign of the one-photon detuning $\Delta$, while the $\chi^{(3)}$ model depends only on $\Delta^{2}$.

Without going into the full calculation of the effect, one may look for a physical reason for the failure of the parametric theory. A clue to the answer is the value of the upper-level detuning, which is smaller in the "bad" case $\left(\delta_{2}=+150\right)$ than in the "good" case $\left(\delta_{2}=-550\right)$. Let us consider now the $\chi^{(5)}$ process shown in Fig. 4: In the bad case, the excitation of the upper-level transition is much closer to resonance than in the good case. Therefore, excess noise due to nonlinear scattering close

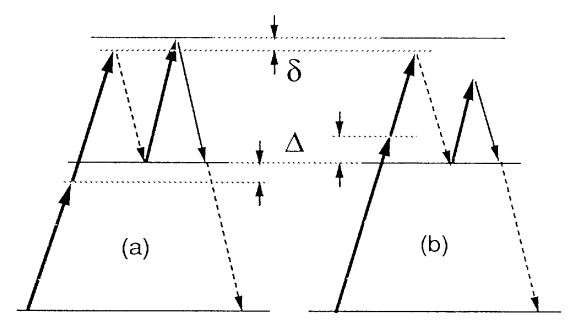

FIG. 4. Diagrams illustrating the two situations discussed in the text. (a) If $\Delta$ and $\delta$ have the same sign and about the same absolute value, the upper transition is excited close to resonance, and strong excess noise occurs. (b) If $\Delta$ and $\delta$ have opposite signs, the excess noise is much smaller. 
to the laser frequency can be expected to be much higher. This process is well known to limit the efficiency of squeezing in two-level systems. ${ }^{24}$ We note that in the case of this three-level system, the excess noise can be greatly reduced by making the right choice for the two detunings $\delta_{1}$ and $\delta_{2}$. The calculation of these effects has been performed, using a more elaborate microscopic description of the nonlinear medium, and will be detailed in other publications. ${ }^{25}$

In conclusion, we have observed a quantum-nondemolition measurement effect using a two-photon nonlinear system. The best reproducibly observed result to date is a reduction of $0.8 \mathrm{~dB}$ below the combined shotnoise levels of the two output beams, which originate from two different lasers. This result is about the same as in previously published experiments in other systems, but is obtained with very small laser powers (typically 1 $\mathrm{mW} \mathrm{cw}$ ). Optimization of the cavity and of the detection system should result in a significant improvement of these values. Moreover, very intriguing predictions can be made in the doubly resonant case, associated with bistable behavior. Therefore, many theoretical and experimental developments can be expected, and threelevel atomic systems seem quite well suited for quantum-noise studies.

We acknowledge fruitful discussions with Serge Reynaud and Jean-Michel Courty. We thank the Commissariat á l'Energie Atomique (Saclay) for the loan of a CR699 dye laser. This work is supported in part by the Direction des Recherches, Etudes et Techniques, and by the Centre National d'Etude des Tèlècommunications. Institut d'Optique is Unité de Recherche Associée 14 of the Centre National de la Recherche Scientifique.

${ }^{1}$ D. Bohm, Quantum Theory (Prentice-Hall, Englewood Cliffs, NJ, 1951).

${ }^{2}$ C. M. Caves, K. S. Thorne, R. W. P. Drever, V. D. Sandberg, and M. Zimmermann, Rev. Mod. Phys. 52, 341 (1980).

${ }^{3}$ B. Yurke, J. Opt. Soc. Am. B 2, 732 (1985).

${ }^{4}$ Y. Yamamoto, N. Imoto, and S. Machida, Phys. Rev. A 33, 3243 (1986).

${ }^{5}$ M. D. Levenson, R. M. Shelby, M. D. Reid, and D. F. Walls, Phys. Rev. Lett. 57, 2473 (1986).

${ }^{6}$ A. LaPorta, R. E. Slusher, and B. Yurke, Phys. Rev. Lett.
62, 28 (1989).

${ }^{7}$ P. Grangier, J. F. Roch, and S. Reynaud, Opt. Commun. 72, 387 (1989).

${ }^{8}$ P. Grangier and J. F. Roch, Quantum Opt. 1, 17 (1989).

${ }^{9}$ H. P. Yuen, Phys. Rev. A 13, 2226 (1976).

${ }^{10}$ L. A. Lugiato and G. Strini, Opt. Commun. 41, 374 (1982).

${ }^{11}$ C. M. Savage and D. F. Walls, Phys. Rev. A 33, 3282 (1986).

${ }^{12}$ B. A. Capron, D. A. Holm, and M. Sargent, III, Phys. Rev. A 35, 3388 (1987).

${ }^{13}$ P. Galatola, L. A. Lugiato, M. Vadacchino, and N. B. Abraham, Opt. Commun. 69, 414 (1989); 69, 419 (1989).

${ }^{14}$ C. A. Blockley and D. F. Walls, Opt. Commun. 79, 241 (1990).

${ }^{15} \mathrm{P}$. Grangier and J. F. Roch, Opt. Commun. (to be published).

${ }^{16}$ The amplifier noise is typically $6 \mathrm{~dB}$ below the observed noise levels, for $0.2-\mathrm{mA}$ photocurrents and $15-\mathrm{MHz}$ analysis frequency. The linearity of the detectors has been checked in the useful range of photocurrents.

${ }^{17}$ Experimentally, we have investigated noise frequencies between 10 and $20 \mathrm{MHz}$, by changing the central frequency of the spectrum analyzer, and modifying accordingly the cavity detuning and the incident ir power, in order to keep the appropriate value of the intracavity power. When the sideband resonance condition is satisfied at $15 \mathrm{MHz}$, the ratio between the intracavity and incident ir powers is about 3. If the laser beam itself resonates, this ratio goes up to about 200 . In this case, the atomic response is saturated and bistability effects can be observed. The resonance of the laser beam itself is therefore avoided during the sweep.

${ }^{18}$ M. D. Levenson and R. M. Shelby, J. Mod. Opt. 34, 775 (1987).

${ }^{19}$ Relative variations of the added and differenced photocurrents can go up to $4 \mathrm{~dB}$, by increasing the atomic density. However, the sodium oven empties very quickly in that case, and associated sub-shot-noise effects have not been observed.

${ }^{20} \mathrm{~J}$. Mertz, A. Heidmann, C. Fabre, E. Giacobino, and S. Reynaud, Phys. Rev. Lett. 64, 2897 (1990).

${ }^{21}$ M. J. Holland, M. J. Collett, D. F. Walls, and M. D. Levenson, Phys. Rev. A 42, 2995 (1990).

${ }^{22}$ R. M. Whitley and C. R. Stroud, Phys. Rev. A 14, 1498 (1976).

${ }^{23}$ S. Reynaud and A. Heidmann, Opt. Commun. 71, 209 (1989).

${ }^{24}$ R. E. Slusher, B. Yurke, P. Grangier, A. LaPorta, D. F. Walls, and M. Reid, J. Opt. Soc. Am. B 4, 1453 (1987).

${ }^{25}$ J. M. Courty, P. Grangier, L. Hilico, and S. Reynaud (to be published). 